\title{
THE RANGE OF A FLEET OF AIRCRAFT*
}

\section{J. N. FRANKLIN $\dagger$}

1. Introduction. The problem discussed in this paper is to determine the range of a fleet of $n$ aircraft with fuel capacities $g_{i}$ gallons and fuel efficiencies $r_{i}$ gallons per mile $(i=1, \cdots, n)$. It is assumed that the aircraft may share fuel in flight and that any of the aircraft may be abandoned at any stage. The range is defined to be the greatest distance which can be attained in this way. Initially the fleet is supposed to have $g$ gallons of fuel.

A theoretical solution is obtained by the method which Richard Bellman [1] calls dynamic programming. Explicit solutions are obtained in the case of two aircraft with different fuel capacities and fuel efficiencies and in the case of any number of aircraft with identical fuel capacities and identical fuel efficiencies.

The problem is similar to the so-called jeep problem. The jeep problem was solved rigorously by N. J. Fine [2]. A solution was also obtained by $\mathrm{O}$. Helmer [3, 4]. Fine cited an unpublished solution by L. Alaoglu. The problem was generalized by C. G. Phipps [5]. Phipps informally developed the special result which is deduced in $\S 4$ of this paper.

2. A recurrence formula. Let $C_{m}$ be any subset of $m$ of the given $n$ aircraft. Mathematically $C_{m}$ may be represented by a subset of $m$ of the first $n$ positive integers. Let $M\left(g, C_{m}\right)$ be defined as the range of the fleet of $m$ aircraft $C_{m}$ starting with $g$ gallons of fuel. Then the required range of the $n$ given aircraft is $M\left(g, C_{n}\right)$, where $C_{n}=\{1,2, \cdots, n\}$. When there is only one aircraft,

$$
M\left(g, C_{1}\right)=\min \left(g / r_{i}, g_{i} / r_{i}\right),
$$

where $C_{1}$ consists of just the $i$ th aircraft.

When there are $m>1$ aircraft, a distance $x$ is flown by all $m$ aircraft. Then one aircraft is abandoned, leaving a subset $C_{m-1} \subset C_{m}$. It is unnecessary to consider abandoning more than one aircraft at a time. For example, the effect of abandoning two aircraft from $C_{m}$ may be obtained by abandoning one of the aircraft from $C_{m}$ and then immediately abandoning the second aircraft from $C_{m-1}$. After the distance $x$ the amount of fuel remaining is

$$
h=g-x \sum_{i \in C_{m}} r_{i} .
$$

The greatest distance which can be attained by the remaining aircraft

* Received by the editors August 20, 1959 and in revised form March 17, 1960.

$\dagger$ California Institute of Technology, Pasadena, California. 
$C_{m-1}$ starting with $h$ gallons of fuel is $M\left(h, C_{m-1}\right)$. If $g, C_{m}$, and $x$ are prescribed, the subset $C_{m-1}$ should be chosen so as to maximize the remaining distance $M\left(h, C_{m-1}\right)$. The total distance traveled will then be

$$
x+\max _{C_{m-1} \subset c_{m}} M\left(g-x \sum_{c_{m}} r_{i}, C_{m-1}\right) .
$$

The maximum distance $M\left(g, C_{m}\right)$ is obtained by maximizing the last expression with respect to $x$. In other words, if $g$ is $\leqq$ the total capacity $\sum g_{i}$ for $i$ in $C_{m}$,

$$
\begin{aligned}
& M\left(g, C_{m}\right) \\
& \quad=\max _{x \leqq g / \Sigma_{C_{m}} r_{i}}\left[x+\max _{C_{m-1} \subset C_{m}} M\left(g-x \sum_{c_{m}} r_{i}, C_{m-1}\right)\right] .
\end{aligned}
$$

In this maximization it is required that $x$ be $\leqq g / \sum r_{i}$, since this is the greatest distance which all $m$ aircraft can fly with $g$ gallons of fuel before one aircraft is abandoned. If $g$ is given in excess of the total fuel capacity $\sum g_{i}$ of the aircraft in $C_{m}$, then some fuel must be thrown away and

$$
M\left(g, C_{m}\right)=M\left(\sum_{c_{m}} g_{i}, C_{m}\right) \quad\left(g>\sum_{c_{m}} g_{i}\right) .
$$

The recurrence formulas (4) and (5) uniquely determine $M\left(g, C_{m}\right)$ for all subsets $C_{m}$ with $m=2,3, \cdots, n$. It is easy to see that each function $M\left(g, C_{m}\right)$ is polygonal in $g$, i.e., continuous and piecewise linear. In fact, (4) may be rewritten, by the identity (2), as

$$
M\left(g, C_{m}\right)=\max _{h \leqq g}\left[a(g-h)+\max _{C_{m-1} \subset c_{m}} M\left(h, C_{m-1}\right)\right],
$$

where $a=1 / \sum_{i \subset c_{m}} r_{i}$. We know from (1) that every function $M\left(g, C_{1}\right)$ is polygonal. Let us suppose that every function $M\left(h, C_{m-1}\right)$ is polygonal. Then

$$
P(h)=\max _{C_{m-1} \subset c_{m}} M\left(h, C_{m-1}\right)
$$

is polygonal, since it is the largest of a finite number of polygonal functions. Now (6) takes the form

$$
M\left(g, C_{m}\right)=a g+\max _{h \leqq g}[-a h+P(h)] .
$$

Since $-a h+P(h)$ is polygonal, its maximum value for $h \leqq g$ is a polygonal function of $g$, say $P^{*}(g)$, and therefore $M=a g+P^{*}(g)$ is also polygonal. This completes an inductive proof that $M\left(g, C_{m}\right)$ is polygonal for $g \leqq \sum g_{i}$, within which range (8) holds. It now follows from (5) that $M\left(g, C_{m}\right)$ is polygonal for all $g$. Incidentally, the identity (8) shows that $M\left(g, C_{m}\right)$ is steadily increasing with rate $\geqq a=1 / \sum_{i \subset c_{m}} r_{i}$ when $g \leqq \sum g_{i}$. For larger values of $g$, (5) shows that $M$ is constant. 
3. The case of two aircraft. Let

$$
M(g)=M\left(g, C_{2}\right), M_{i}(g)=M\left(g, C_{1}\right) \quad\left(C_{1}=i=1,2\right) .
$$

For $g \leqq g_{1}+g_{2}$, (8) takes the form

$$
M(g)=a g+\max _{h \leqq g}[-a h+P(h)],
$$

where

$$
P(h)=\max _{i=1,2} M_{i}(h), \quad a=1 /\left(r_{1}+r_{2}\right) .
$$

By the identity (1),

$$
M_{i}(h)=\min \left(h / r_{i}, g_{i} / r_{i}\right) \quad(i=1,2) .
$$

Without loss of generality it will be assumed that $r_{1} \leqq r_{2}$.

Case 1. Suppose that $g_{1} / r_{1} \geqq g_{2} / r_{2}$. In this case

$$
P(h)=M_{1}(h)
$$

(for all $h)$.

Then

$$
-a h+P(h)= \begin{cases}-a h+h / r_{1} & \left(h \leqq g_{1}\right) \\ -a h+g_{1} / r_{1} & \left(h \geqq g_{1}\right) .\end{cases}
$$

Since $a<1 / r_{1}$, it follows that

$$
P^{*}(g)=\left\{\begin{array}{lc}
-a g+g / r_{1} & \left(g \leqq g_{1}\right) \\
-a g_{1}+g_{1} / r_{1} & \left(g \geqq g_{1}\right),
\end{array}\right.
$$

where $P^{*}(g)=\max [-a h+P(h)]$ for $h \leqq g$. From (8) it follows that

$$
M(g)= \begin{cases}g / r_{1} & \left(g \leqq g_{1}\right) \\ a\left(g-g_{1}\right)+g_{1} / r_{1} & \left(g_{1} \leqq g \leqq g_{1}+g_{2}\right) .\end{cases}
$$

For $g>g_{1}+g_{2}$, equation (5) gives

$$
M(g)=a g_{2}+g_{1} / r_{1} \quad\left(g>g_{1}+g_{2}\right) .
$$

From the definition (2) of the remaining fuel $h$ as a function of the distance $x$ to be traveled by both aircraft, it is clear that the optimal procedure in Case I is to use just aircraft 1 if $g \leqq g_{1}$, or if $g>g_{1}$ to use both aircraft until only $g_{1}$ gallons of fuel remain and then to complete the trip with just aircraft 1.

Case 2. Suppose that $g_{1} / r_{1}<g_{2} / r_{2}$. In this case

$$
P(h)= \begin{cases}h / r_{1} & \left(h \leqq g_{1}\right) \\ g_{1} / r_{1} & \left(g_{1} \leqq h \leqq g^{*}\right) \\ h / r_{2} & \left(g^{*} \leqq h \leqq g_{2}\right) \\ g_{2} / r_{2} & \left(h \leqq g_{2}\right)\end{cases}
$$


where $g^{*}=r_{2} g_{1} / r_{1}$ is the abscissa of the point of intersection of the graphs of $M_{1}(h)$ and $M_{2}(h)$. The function $-a h+P(h)$ is a polygonal function with peaks at $h=g_{1}$ and $h=g_{2}$. There are two subcases, depending upon whether the first peak is higher (A) or lower (B) than the second peak.

Case 2A. Suppose that $g_{1} / r_{1}<g_{2} / r_{2}$ and $g_{1} / r_{1}{ }^{2} \geqq g_{2} / r_{2}{ }^{2}$. Then

$$
P^{*}(g)=\left\{\begin{array}{lc}
-a g+g / r_{1} & \left(g \leqq g_{1}\right) \\
-a g_{1}+g_{1} / r_{1} & \left(g \geqq g_{1}\right),
\end{array}\right.
$$

and, as in Case 1, $M(g)$ has the form (16), (17). The optimal procedure in this case is the same as that in Case 1.

Case $2 B$. Suppose that $g_{1} / r_{1}<g_{2} / r_{2}$ and $g_{1} / r_{1}{ }^{2}<g_{2} / r_{2}{ }^{2}$. Let $g^{\prime}=g_{1} r_{2}{ }^{2} / r_{1}{ }^{2}$; this is the first value of $h>g_{1}$ at which $-a h+P(h)=-a g_{1}+P\left(g_{1}\right)$. Then

$$
P^{*}(g)= \begin{cases}-a g+g / r_{1} & \left(g \leqq g_{1}\right) \\ -a g_{1}+g_{1} / r_{1} & \left(g_{1} \leqq g \leqq g^{\prime}\right) \\ -a g+g / r_{2} & \left(g^{\prime} \leqq g \leqq g_{2}\right) \\ -a g_{2}+g_{2} / r_{2} & \left(g \leqq g_{2}\right)\end{cases}
$$

Therefore,

$$
M(g)= \begin{cases}g / r_{1} & \left(g \leqq g_{1}\right) \\ a\left(g-g_{1}\right)+g_{1} / r_{1} & \left(g_{1} \leqq g \leqq g^{\prime}\right) \\ g / r_{2} & \left(g^{\prime} \leqq g \leqq g_{2}\right) \\ a\left(g-g_{2}\right)+g_{2} / r_{2} & \left(g_{2} \leqq g \leqq g_{1}+g_{2}\right)\end{cases}
$$

and, according to equation (5),

$$
M(g)=a g_{1}+g_{2} / r_{2} \quad\left(g>g_{1}+g_{2}\right)
$$

The optimal procedure is as follows. If $g \leqq g_{1}$, use only aircraft 1 . If $g_{1} \leqq g \leqq g^{\prime}$, use both aircraft until only $g_{1}$ gallons remain; then use just aircraft 1 . If $g^{\prime} \leqq g \leqq g_{2}$, use only aircraft 2 . If $g_{2} \leqq g \leqq g_{1}+g_{2}$, use both aircraft until only $g_{2}$ gallons remain; then use just aircraft 2 . If $g$ $>g_{1}+g_{2}$, some fuel must be thrown away, and the trip is made with $g=g_{1}+g_{2}$ as described in the preceding sentence.

From these results it is apparent that in the general case of $n$ aircraft the optimal policy will depend in a complicated way upon $g$ as well as upon the $g_{i}$ and $r_{i}$. For example, the value of $g$ may determine which of the aircraft finishes the trip. 


\section{The case of identical aircraft. Let}

$$
g_{i}=G, \quad r_{i}=R \quad(i=1, \cdots, n),
$$

and let $M_{m}(g)=M\left(g, C_{m}\right)(m=1, \cdots, n)$. In this case the recurrence formulas (4), (5) take the form

$$
\begin{array}{cc}
M_{m}(g)=\max _{x \leqq g / m R}\left[x+M_{m-1}(g-m R x)\right] & (g \leqq m G), \\
M_{m}(g)=M_{m}(m G) & (g>m G) .
\end{array}
$$

It will be shown that, if $k=[g / G]$, the greatest integer $\leqq g / G$,

$$
\begin{array}{lrl}
M_{m}(g)=\frac{G}{R}\left(1+\frac{1}{2}+\cdots+\frac{1}{k}\right)+\frac{g-k G}{(k+1) R} & (g \leqq m G, k \geqq 1), \\
M_{m}(g)=\frac{G}{R}\left(1+\frac{1}{2}+\cdots+\frac{1}{m}\right) & (g>m G) .
\end{array}
$$

The right-hand side of (26) is defined as $g / R$ when $k=0$. In the optimal policy, if $g=k G \leqq m G$, the trip is begun with $k$ aircraft. If $k G<g$ $<(k+1) G \leqq m G$, the trip is begun with $k+1$ aircraft. If $g>m G$, then $g-m G$ gallons of fuel must be thrown away, and the trip is begun with all $m$ aircraft. In any case, if the trip is begun with $K$ aircraft, the first aircraft is abandoned when only $(K-1) G$ gallons of fuel remain. Then $K-1$ aircraft are flown until only $(K-2) G$ gallons remain, and so on.

This result can be established by induction. If $m=1$, formulas (26) and (27) become

$$
\begin{aligned}
& M_{1}(g)=g / R \quad(g \leqq G), \\
& M_{1}(g)=G / R \quad(g>G),
\end{aligned}
$$

which is correct according to (1). Assume that the result holds for $m-1$ aircraft. Then

$$
\frac{d}{d h} M_{m-1}(h) \geqq \frac{1}{(m-1) R} \quad(0<h<(m-1) G)
$$

at all points $h$ at which the polygonal function $M_{m-1}(h)$ has a derivative. Therefore, $x+M_{m-1}(g-m R x)$ is a steadily decreasing function of $x$ for $0 \leqq x \leqq g / m R$ if $g \leqq(m-1) R$. Setting $x=0$ in (24) gives the maximum value

$$
M_{m}(g)=M_{m-1}(g) \quad(g \leqq(m-1) G) .
$$

But $M_{m-1}(g)$ is given by the right-hand side of $(26)$ for all $g \leqq(m-1) G$. Therefore, $(26)$ is established for $g \leqq(m-1) G$.

Next suppose that $(m-1) G<g \leqq m G$. Then $M_{m-1}(g-m R x)$ is constant for $g-m R x \geqq(m-1) G$; for larger values of $x$ the rate of in- 
crease is $\leqq-m R /(m-1) R<-1$. Therefore, the maximum (24) is attained when $g-m R x=(m-1) G$, and

$$
\begin{aligned}
M_{m}(g) & =\frac{g-(m-1) G}{m R}+M_{m-1}((m-1) G) \\
& =\frac{g-(m-1) G}{m R}+\frac{G}{R}\left(1+\frac{1}{2}+\cdots+\frac{1}{m-1}\right) .
\end{aligned}
$$

This establishes the result $(26)$ for $(m-1) G<g \leqq m G$. The result (27) for $g>m G$ follows from (5).

5. An asymptotic formula for $\mathrm{g}$. The solution (26), (27) in the case of identical aircraft is similar to the solution of the jeep problem, although the solutions were established by different methods. In this section an asymptotic formula will be developed for the amount of fuel $g$ which is necessary in order to transport identical aircraft a distance $x$. Let $g=f(x)$. It will be shown that

$$
f(x)=A(x)+O(\exp (-R x / G)),
$$

where

$$
A(x)=G\left(-\frac{1}{2}+\exp \left(\frac{R x}{G}-C\right)\right) .
$$

In these identities $G$ and $R$ are the fuel capacity and the fuel efficiency of

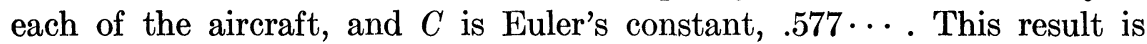
comparable to Fine's asymptotic formula for the solution of the jeep problem [2].

From the result of the last section it is clear that $x$ is the range of $n+1$ aircraft with initial fuel supply $g$, where $n G<g \leqq(n+1) G$. Setting $m=n+1$ in $(26)$ gives

$$
x=x^{*}+\frac{g-n G}{(n+1) R},
$$

where

$$
\begin{aligned}
x^{*} & =\frac{G}{R}\left(1+\frac{1}{2}+\cdots+\frac{1}{n}\right) \\
& =\frac{G}{R}\left(\log n+C+\frac{1}{2 n}+O\left(\frac{1}{n^{2}}\right)\right) .
\end{aligned}
$$

This well-known asymptotic formula is derived in [6, p. 529]. From (36) it follows that

$$
\exp \left(\frac{R x^{*}}{G}-C\right)=n \exp \left(\frac{1}{2 n}+O\left(\frac{1}{n^{2}}\right)\right)=n+\frac{1}{2}+O\left(\frac{1}{n}\right) .
$$


This relation shows that

$$
n \geqq \text { constant }+\exp \left(\frac{R x^{*}}{G}-C\right),
$$$$
\frac{1}{n}=O\left(\exp \left(-R x^{*} / G\right),\right.
$$

$$
n=-\frac{1}{2}+\exp \left(\frac{R x^{*}}{G}-C\right)+O\left(\exp \left(-R x^{*} / G\right)\right) .
$$

Since $n G=f\left(x^{*}\right)$, multiplication by $G$ gives

$$
f\left(x^{*}\right)=A\left(x^{*}\right)+O\left(\exp \left(-R x^{*} / G\right)\right) .
$$

In order to justify replacement of $x^{*}$ by $x$ in the identity (41), it is convenient first to show that

$$
f^{\prime}(x)-A^{\prime}(x)=O(1) \quad\left(0<x-x^{*}<G /(n+1) R\right) .
$$

Differentiation of $g$ as a function of $x$ in (35) gives

$$
f^{\prime}(x)=(n+1) R .
$$

But

$$
\begin{aligned}
& A^{\prime}\left(x^{*}\right)<A^{\prime}(x)=R \exp \left(\frac{R x}{G}-C\right) \\
& \quad \leqq A^{\prime}\left(x^{*}+G /(n+1) R\right) \leqq A^{\prime}\left(x^{*}\right) \exp (1 /(n+1)) .
\end{aligned}
$$

Since, by $(39), A^{\prime}\left(x^{*}\right) /(n+1)=O(1)$, it follows that

$$
A^{\prime}(x)=A^{\prime}\left(x^{*}\right)+O(1) .
$$

Subtraction of (45) from (43) gives

(46) $f^{\prime}(x)-A^{\prime}(x)=(n+1) R-R \exp \left(\frac{R x^{*}}{G}-C\right)+O(1)$.

The required relation (42) now follows from (40). Integration of (42) gives

$$
\begin{aligned}
& f(x)-A(x)=f\left(x^{*}\right)-A\left(x^{*}\right)+O\left(x-x^{*}\right) \\
& =O\left(\exp \left(-R x^{*} / G\right)\right)+O(1 / n)=O\left(\exp \left(-R x^{*} / G\right)\right) .
\end{aligned}
$$

Since $x-x^{*}$ is bounded, $x^{*}$ may be replaced by $x$ in the last expression, and this gives the asymptotic formula (33). 


\section{REFERENCES}

1. Richard Bellman, Dynamic Programming, Princeton University Press, Princeton, 1957.

2. N. J. Fine, The jeep problem, Amer. Math. Monthly, 54 (1947), pp. 24-31.

3. O. Helmer, A problem in logistics: The jeep problem, RAND Report RA-15015, 1946.

4. O. Helmer, A problem in logistics: The jeep problem (Part 2), RAND Report RA-15019, 1947.

5. C. G. Phipps, The jeep problem: A more general solution, Amer. Math. Monthly, 54 (1947), pp. 458-462.

6. Konrad Knopp, Theory and Application of Infinite Series, Blackie and Son, London, 1949. 